\title{
Constant Sustainable Consumption Rate in Optimal Growth with Exhaustible Resources*
}

\author{
By Frederic Y. M. Wan†
}

The problem of optimal growth with an exhaustible resource deposit under R. M. Solow's criterion of maximum sustainable consumption rate, previously formulated as a minimum-resource-extraction problem, is shown to be a Mayer-type optimal-control problem. The exact solution of the relevant firstorder necessary conditions for optimality is derived for a Cobb-Douglas production function, whether or not the constant unit resource extraction cost vanishes. The related problem of maximizing the terminal capital stock over an unspecified finite planning period is investigated for the development of more efficient numerical schemes for the solution of multigrade-resource deposit problems. The results for this finite-horizon planning problem are also important from a theoretical viewpoint, since they elucidate the economic content of the optimal growth paths for infinite-horizon problems.

\section{Introduction}

Social justice and our insatiable appetite for the earth's finite stock of nonrenewable resources, such as fossil fuel and minerals, have been two of the major societal concerns of the seventies. Even a casual reading of the 1974 volume of The Review of Economic Studies alone would convince any skeptics that there has been a revived interest in the economics of exhaustible resources, at least among the social scientists, particularly in the area of optimal economic growth and capital accumulation in the presence of a finite stock of nonrenewable

Address for correspondence: Professor F. Y. M. Wan, Institute of Applied Mathematics and Statistics, The University of British Columbia, Vancouver, B.C. V6T 1W5 Canada.

*This research was supported by National Research Council Grant No. A9259. The content of this paper was presented at the 1977 A.M.S. Summer Meeting in Seattle, Washington.

+Professor of Mathematics \& Director of the Institute of Applied Mathematics and Statistics (I.A.M.S.). 
resources which are essential to the production of consumption goods. To those concerned with social justice, the optimal growth research results should be rather unsettling. With the conventional social rate of time preference in favor of the present and with "the drag exercised by the essential but exhaustible resources" [1], it is possible that the optimal growth for an infinite horizon will lead to a consumption per head which tends to zero asymptotically with increasing time. The fact that the optimal growth path might dictate eventual extinction is a direct consequence of the conventional utilitarian approach to social welfare, defined as the sum of the welfares or utilities of individual members of the society', which allows for the possibility that a loss of utility to one or more members can be more than offset by an increment to others. As long as the destined-to-become-defunct last generation cannot be present to defend its own interest, it behooves us who control its destiny to reexamine and perhaps reformulate our concept of and approach to optimal growth so that the earth's nonrenewable resources may be exploited in a manner that is fair to future generations, at least in the context of growth theory.

In A Theory of Justice [2], John Rawls argues in effect that inequality in the distribution of wealth or utility is justified only if it is a necessary condition for the improvement of the poorest member. This concept of social welfare, which Rawls proposes for contemporary members of a society (i.e., intrageneration), was formulated as a mathematical statement in the form of a max-mm principle by R. M. Solow in [3]; he then studied its implications for optimal growth and intergenerational equity in [3], and later with the present author in [4]. With the max-min principle as a (new) criterion for optimal growth, Solow deduced that the consumption per head must be the same for all generations, and the determination of the optimal growth path amounts to finding a time path for extracting the finite stock of resources which maximizes the constant level of consumption per head [3]. At the time, this optimization problem appeared to be a rather unconventional one and not amenable to a direct method of solution. An indirect method developed in [3] and used also in [4] consists of minimizing the total resources used for a fixed consumption level and then adjusting the consumption level so that the corresponding optimal resource extraction policy exhausts the resource stock over the planning period. This alternate formulation gave rise to a set of necessary conditions for the optimal growth path of the intuitively equivalent problem. Even for simplest but still informative case, the solution of the optimal growth path was obtained by direct verification of an "educated guess" [3]. An elaborate and time consuming search scheme had to be developed to handle more general problems numerically (see [4] for example).

In this article, we show that various optimal-growth problems under the max-min criterion may be formulated as Mayer-type problems, and therefore first-order necessary conditions for their solution in the form of a boundaryvalue problem for a system of nonlinear ordinary differential equations may be

\footnotetext{
${ }^{1}$ In the context of economic growth theory, a member of the society means a whole generation, or more precisely, the entire population at a moment in time, since individual persons are usually taken as identical and treated identically in such a theory.
} 
obtained by conventional methods of optimal control theory [5]. ${ }^{2}$ We also show that the exact solution of the relevant boundary-value problems, including those studied in [3] and [4], can be obtained by an efficient deductive procedure.

After reformulating the optimal-growth problem of [3] (maximum constant consumption rate per head) as a Mayer problem in Sec. 3, the first-order necessary conditions for an optimal trajectory of the finite-horizon version of the problem will be obtained from the maximum principle. With these first-order necessary conditions from the maximum principle assumed to hold also for the infinite-horizon case, these conditions are effectively the same as those obtained in [3] for the minimum-resource-extraction problem. We deduce in Sec. 4 the exact solution of the revelant boundary-value problem associated with these necessary conditions for a single-grade resource deposit in the case of a CobbDouglas production function with a nonzero extraction cost. This solution reduces to that obtained in [3] for the case of no extraction cost.

With a view toward a more efficient and accurate solution scheme for the two-grade resource problem studied in [4], we formulate in Sec. 5 the problem of maximizing the capital-stock accumulation at the end of a finite planning period for a fixed constant consumption rate per head. The exact solution of the boundary-value problem defined by the first-order necessary conditions for optimality for this problem will be obtained, again for a Cobb-Douglas production function. The synthesis of the results in Secs. 4 and 5 for an efficient algorithm for the numerical solution of the two-grade deposit problem will be discussed in Sec. 6. The results for the finite-horizon planning problem also elucidate the economic content of the optimal growth paths of related infinitehorizon planning problems.

\section{The economic model}

In the simple economic model studied in [3] and [4], there is a single commodity produced under constant returns to scale according to a well-defined technology whose inputs are the services of capital and labor and a certain amount of a single nonrenewable and nonaugmentable resource. Population and employment are constant, so we might as well use a labor intensive (i.e., per head) description of the mathematical model. In particular, if $q$ is the output of the commodity per head (net of depreciation) at any instant t, then we have $\mathrm{q}=f(k, r)$, where $\boldsymbol{k}$ and $\mathrm{r}$ are the capital stock per head and resource flow per head, respectively, and where $f$ is a well-behaved neoclassical production function with $f(0, r)=f(k, 0)=$ 0 , so that the inputs are "essential" to the production. (With constant returns to scale, labor does not appear explicitly in a per-head description.) The commodity produced is used for immediate consumption, for payment of the cost of extracting the resource and for investment to increase the existing stock of capital, so that

$$
4=c+\dot{k}+\theta r, \quad \text { or } \dot{k}=f(k, r)-\theta r-c,
$$

\footnotetext{
${ }^{2}$ Thenecessary conditions for cases involving an infinite planning period are formal results, since we do not have a maximum principle for these cases. However, the supporting evidence indicates that they lead to the correct optimal growth path.
} 
where a dot indicates differentiation with respect to time $\mathrm{t}, \mathrm{c}$ is the consumption rate per head, and 8 is the unit extraction cost of the resource (taken to be a known constant in this study if not otherwise stated).

At any instant, the economy is characterized by the stock of capital it has inherited from the past and the remains of an initial (finite) deposit, $\boldsymbol{D}_{\mathbf{0}}$, of the nonrenewable and nonaugmentable resource. The economy must choose how much of the natural resource to mine and use currently, so that $\mathrm{r}(\mathrm{t})$ is a decision variable. Once decided, the current output is determined by $q=f(k, r)$ and an amount equal to $\boldsymbol{q}-\boldsymbol{\theta} \boldsymbol{r}$ is available for consumption and investment. The economy must then decide the allocation of output between those two uses. The allocation of net investment determines the stock of capital for the next instant, and the decision process described above repeats itself. The problem for society is to make its decisions in such a way that the resulting time path of the economy will be optimal under a stipulated criterion. The possible time paths are limited by the initial capital stock $\boldsymbol{k}_{0}$, the fact that $\boldsymbol{k}(\boldsymbol{t})$ and $\mathrm{r}(\mathrm{t})$ cannot be negative and the finiteness of the resource deposit. In particular, we must have, over the planning period $T$,

$$
\int_{0}^{T} r(t) d t=D_{0}
$$

(Strictly speaking, the left hand side of (2.2) may be less than or equal to $D_{0}$; but a program cannot be optimal if it does not completely use up the resource deposit. ${ }^{3}$ )

The criterion of optimality used in this study will be the maximization of a permanently sustainable (constant) level of consumption per head, first proposed in [3] and further studied in [4]. Under this criterion, the consumption rate per head, c [see Eq. (2.1)], is stipulated to be a constant, and an extraction rate $\mathrm{r}(\mathrm{t})$ is sought so that the associated economy allows the largest possible value of c. If there is no future beyond a single finite planning period, we stipulate $\boldsymbol{k}(\boldsymbol{T})=\boldsymbol{0}$.

To summarize, our problem is to choose $\mathrm{r}(\mathrm{t})$ to maximize the constant consumption rate per head $\mathrm{c}$ subject to the equation for capital accumulation (2.1) in which c appears, a prescribed initial stock of capital,

$$
k(0)=k_{0}
$$

the nonnegativity constraints

$$
r(t) \geqslant 0 \text { and } k(t) \geqslant 0
$$

and the condition of a finite nonrenewable and nonaugmentable resource deposit expressed by (2.2) For the case of a finite planning period, we have the additional stipulation of a terminal capital stock,

$$
k(T)=k_{T}
$$

${ }^{3}$ For any $\mathbf{r}>0$, we assume $\partial f / \partial r>\theta$, for sufficiently large positive $k$, which is the case for the Cobb-Douglas production function used in this paper and in [3] and [4]. 
with $\boldsymbol{k}_{\boldsymbol{T}}=\boldsymbol{O}$ if there is no future beyond the current planning period. Such a terminal condition on the capital stock is absent for the infinite-horizon case.

\section{A problem of $M$ ayer}

To transform the rather unconventional optimization problem described in Sec. 2 into a standard optimal-control problem, we introduce a new state variable $\mathrm{D}(\mathrm{t})$ by the differential equation

$$
\dot{D}=-r
$$

with

$$
\mathrm{D}(0)=D_{0}
$$

In other words, $\mathrm{D}(\mathrm{t})$ is the remaining amount of resource deposit at time $t$. The condition (2.2) then becomes

$$
\mathrm{D}(\mathrm{T})=0 .
$$

Next, we introduce the stipulation of a constant $\mathrm{c}$ indirectly by thinking of $\mathrm{c}$ as another state variable with an equation of state

$$
\dot{\boldsymbol{c}}=0 .
$$

The optimization problem of Sec. 2 may then be stated as one of choosing $r(t)$ to maximize the terminal value of $c(t)$, namely $c(T)$, subject to the equations of state (2.1), (3.1) and (3.3), the boundary conditions (2.3), (3.2a, b) and, if $\boldsymbol{T}<\infty$, (2.9, and the nonnegativity conditions (2.4). In this new formulation, our optimization is in the form of a standard Mayer type optimal control problem. The first-order necessary conditions for an optimal trajectory for such a problem can be expressed in terms of three costate variables (or Lagrange multipliers). They consist of three first-order ODE

$$
\begin{aligned}
-\dot{\lambda}_{k} & =\lambda_{k} \frac{\partial f}{\partial k}, \\
-\dot{\lambda}_{D} & =0, \\
\dot{\lambda}_{c} & =\lambda_{k} ;
\end{aligned}
$$

the inequality

$$
\frac{\partial H}{\partial r}>0, \quad H \equiv \lambda_{k}[f-\theta r-c]+\lambda_{D}[-r]+\lambda_{c}[0],
$$


for $0<t<\mathrm{T}$; and the transversality conditions

$$
\lambda_{c}(0)=0 \text { and } \lambda_{c}(T)=1
$$

at the end points. For the infinite-horizon case, we assume that these first-order necessary conditions continue to hold with $T=\infty$ and, in the absence of a terminal condition on the capital stock, there is the additional transversality condition

$$
\lim _{t \rightarrow \infty} \lambda_{k}(t)=0
$$

When the equality holds for (3.7) and the constraints (2.4) are not binding, Eqs. (2.1), (3.1), (3.3), (3.4)-(3.7) together with the boundary conditions (2.3), (3.2), (3.8) and (2.5) [or (3.9) for the infinite-horizon case] constitute a two-point boundary-value problem for a sixth-order system of ODE involving seven unknown functions, $k, r, c, D, \lambda_{k}, \lambda_{D}$ and $\lambda_{c}$.

The costate variables $\lambda_{k}, \lambda_{D}$ and $\lambda_{c}$ are the shadow price of capital, the resource and the consumption rate, respectively, and the economic interpretation of (3.5) and (3.6) is clear. In fact, we have from (3.5) a constant shadow price of the resource, i.e., $\lambda_{D}=$ constant. Let

$$
p(t)=\lambda_{k}(t) / \lambda_{D}
$$

and write the condition (3.4), (3.7) as

$$
\frac{\dot{p}}{p}=-\frac{\partial f}{\partial k}, \quad p\left(\frac{\partial f}{\partial r}-\theta\right) \geqslant 1
$$

When $8=0$ and the equality holds in $(3.1 \mathrm{lb})$, the above two equations are the same as the first-order necessary conditions obtained in [3] for the minimumresource-extraction problem. For $\boldsymbol{\theta} \neq 0$, the economic content of (the equality) (3.1 lb) is simply that the resource should be drawn down in such a way that its net (of extraction cost) marginal value product is kept to its own shadow price in terms of the shadow price of capital, $1 / p$. The condition $(3.1$ la), giving the rate of change of $\mathrm{p}$ (or $1 / \mathrm{p})$, is identical to the necessary condition $(9 \mathrm{~b})$ for the minimum-resource-extraction problem of [3], independent of the resource extraction cost. Therefore, its economic interpretation is exactly the same as that for (9b) of [3], leading to the observation that, along the optimal growth path, "a rational investor calculating with efficiency prices should be at all times indifferent at the margin between holding capital goods and holding resource deposit as earning assets"[3].

It having served a useful purpose in an optimal-control formulation of our optimization problem, we can now discard the trivial ODE (3.2) from the solution process and simply take $\mathrm{c}$ to be a constant for all time. With the equality holding in (3.7) and the constraints (2.4) not binding, we have then a fourth-order system for the five unknown functions $k(t), r(t), D(t), p(t)\left[\right.$ or $\left.\lambda_{k}(t)\right]$ 
and $\boldsymbol{\lambda}_{\boldsymbol{c}}$ with $\boldsymbol{\lambda}_{\boldsymbol{D}}$ and c as unknown parameters. The system is supplemented by a set of six boundary conditions, three at each of the two end points of the planning period. The fourth-order system uncouples into three subsystems. The second-order system (2.1), (3.1 la), (3.11 b) (with equality holding), supplemented by the auxiliary conditions (2.3) and (2.5) or (3.9), determines $k(t ; \mathrm{c}), \mathrm{r}(\mathrm{t} ; \mathrm{c})$ and $\mathbf{p}(t ; c)$ with $\mathrm{c}$ as an unknown parameter. The first-order ODE (3.1) and the two auxiliary conditions $(3.2 \mathrm{a}, \mathrm{b})$ determine $\mathrm{D}(\mathrm{t})$ and $\mathrm{c}$. Finally, we may if we wish use the first-order ODE (3.6), or

$$
\dot{\lambda}_{c}=\lambda_{D} p
$$

and the two auxiliary conditions (3.8a, b) to determine $\lambda_{c}(t)$ and the constant $\lambda_{D}$. It is not difficult to verify that for the infinite-horizon case, the first two of these subproblems are equivalent to the governing two-point boundary-value problem in [3] and [4] for the determination of the optimal growth path of the minimumresource-extraction problem. We do this in the next section for the case of a Cobb-Douglas production function treated in [3] and [4] and show that the exact solution of the boundary-value problem for the case $\theta=0$ obtained in [3] can be deduced by a systematic procedure and that the same procedure also yields an exact solution for the $\boldsymbol{\theta} \neq 0$ case not known hitherto.

\section{A Cobb-D ouglas production function}

To compare with the results in [3], we now study the solution of the fourth-order boundary-value problem obtained in Sec. 3 for the Cobb-Douglas production function $f=f_{0} k^{a} r^{b}$, where $f_{0}, a$ and $b$ are known positive constants with $0<a+b$ $<1$ and $0<\mathrm{b}<a$. For this case, Eq. (2.1) becomes

$$
\dot{k}=f_{0} k^{a} r^{b}-\theta r-c .
$$

For an interior solution of the optimal-control problem, i.e., when equality holds in (3.7), Eqs. (3.7) and (3.4) [or (3.1 la, b)] become

$$
p\left(f_{0} b k^{a} r^{b-1}-\theta\right)=1
$$

and

$$
\dot{p}=-p f_{0} a k^{a-1} r^{b},
$$

respectively, where $\mathrm{p} \equiv \lambda_{k} / \lambda_{D}$. Upon differentiating both sides of (4.2) and using (4.1) and (4.3) to eliminate $\dot{k}$ and $\dot{\boldsymbol{p}}$ from the resulting equation, we get

$$
\dot{r}=\frac{a c r}{(1-b) k}\left[\frac{\theta(1-b)}{b c} r-1\right]
$$


For $\theta=0$, the ODE (4.1) and (4.4) are the same as the governing equations of 3 ] for the minimum-resource-extraction problem.

For a constant $\theta$ not necessarily zero, the pair (4.1) and (4.4) admits an exact solution in terms of elementary functions, since

$$
\frac{d k}{d r}=\xi(r) k^{a+1}-\eta(r) k
$$

where

$$
\xi(r)=\frac{1-b}{a c} \frac{f_{0} r^{b-1}}{\bar{\theta} r-1}, \quad \eta(r)=\frac{1-b}{a c r} \frac{\theta r+c}{\bar{\theta} r-1}
$$

and $\overline{\boldsymbol{\theta}}=\boldsymbol{\theta}(\mathbf{1}-\boldsymbol{b}) / \boldsymbol{b c}$, is a Bernoulli-type equation. For the infinite-horizon case investigated in [3] and [4], the exact solution of (4.5) subject to the transversality condition (3.9) written as

$$
\lim _{t \rightarrow \infty} \mathrm{p}(\mathrm{t})=0
$$

where $p(t)$ is given in terms of $r$ and $k$ by (4.2), is ${ }^{4}$

$$
f_{0}(1-b) k^{a} r^{b}=c
$$

with

$$
p(t)=\frac{1-b}{b c} \frac{r}{1-\bar{\theta} r}
$$

For a given c, the (formal) necessary conditions for optimality for the infinite-horizon case specify nonnegative-time paths for the capital accumulation and resource extraction rate which correspond to a unique trajectory (4.8) in the first quadrant of the k,r-plane. This unique "optimal trajectory" is independent of $\theta$. That is, whatever the unit extraction cost $\theta$ may be, the output is the same for all time, and at any instant the same fraction $(1-b)$ of output is consumed and the rest is for extraction cost and investment. Of course, the actual amount of output (and therefore the consumption rate per head) decreases with increasing. $\boldsymbol{\theta}$, as we shall see. For a given initial capital stock, we must choose

$$
r(0)=r_{0} \equiv\left[\bar{f}_{0} k_{0}^{a}\right]^{-1 / b}, \quad \bar{f}_{0} \equiv(1-b) f_{0} / c .
$$

The calculation is simplified considerably by using the fact that, for a finite resource deposit, we must have $r \rightarrow 0$ as $t \rightarrow \infty$ [which implies $p(\infty)=0$ and vice versa]. 
to get on the "optimal trajectory" (4.8). A more thorough discussion of trajectories in the k,r-plane can be found in the Appendix of this paper.

We now use the relation (4.8) to eliminate $\boldsymbol{k}$ from (4.4) to get a first-order ODE for $r$ alone:

$$
\dot{r}=-\underset{1-b}{\operatorname{ac}}\left[\bar{f}_{0} r^{(a+b)}\right]^{1 / a}[1-\bar{\theta} r]
$$

with $\bar{f}_{0}=(1-b) f_{0} / \boldsymbol{c}$ and $\bar{\theta}=\theta(1-b) / b c$ as previously defined. Equation (4.11) is separable and, subject to the initial condition (4. 10), can be solved to get $\mathrm{r}(\mathrm{t} ; \mathrm{c})$. The unknown parameter $\mathrm{c}$ is then determined by the boundary-value problem for $\mathrm{D}(\mathrm{t})$ defined by (3.1) and (3.2). The solution of this problem is

$$
D(t ; c)=\int_{t}^{\infty} r(t ; c) d t
$$

with

$$
D(0 ; c)=\int_{0}^{\infty} r(t ; c) d t=D_{0}
$$

For the special case $8=0$ investigated in [3], we get from (4.11) and (4. 10) the unique solution

$$
r(t)=\left|\frac{\mathrm{c}}{f_{1}(1-b)\left\{k_{0}+\frac{b c t}{l-b}\right\}^{a}}\right|^{1 / b},
$$

and then from (4.8)

$$
k(t)=k_{0}+\frac{b c t}{1-b}
$$

with c determined by (4.13) to be

$$
c=(1-b)\left\{f_{0} k_{0}^{a-b} D_{0}^{b}(a-b)^{b}\right\}^{1 /(1-b)} \equiv c_{\max }
$$

The expressions (4.14), (4.15) and (4.16) are the same as those obtained in[3] for the minimum-resource-extraction problem. From (4.12), we have also

$$
D(t ; c)=\frac{1}{a-b}\left[\frac{1}{f_{0}}\left(\frac{c}{1-b}\right)^{1-b}\left(k_{0}+\frac{b c t}{1-b}\right)^{b-a}\right]^{1 / b}
$$


which will be useful in later development. We omit the solution for $\lambda_{c}(t)$ and $\lambda_{D}$ [which can be obtained from (3.6) and (3.8)], as these quantities are not pertinent to our discussion. It should be noted that the (continuously differentiable) solution of the BVP which summarizes the (formal) necessary conditions for optimality is unique; therefore, it is the (formal) optimal solution.

For the more general case of a single-grade resource deposit with a nonzero (constant) extraction cost $(\theta \neq 0)$ not previously investigated, the exact solution of the initial-value problem for $r(t)$ defined by (4.11) and (4. 10), is in the form of a quadrature giving $\mathrm{i}$ in terms of $r$. Consequently, $c$ is determined numerically by the conventional shooting method, which solves the IVP (4.11), (4. 10), (3.1), (3.2a) numerically for a particular value of $c$ and then iterates on c (by the secant method) until (3.2b) is satisfied. To implement such a numerical scheme, it is important to note that c must be sufficiently small so that

$$
\theta r_{0}=\frac{\theta}{b}\left[\left.\frac{c^{1-b}}{f_{0} k_{0}^{a}(1-b)^{1-b}}\right|^{1 / b}<1\right.
$$

Otherwise, $r(t)$ would be a monotone increasing function of time [see Eq. (4.11)] and the entire finite stock of resource would be exhausted in finite time. Also for the numerical scheme, the infinite horizon is replaced by a large but finite interval $(0, \bar{T})$, and the condition (3.2b) at infinity is replaced by the requirement that the numerically generated $\mathrm{D}(\bar{T})$ be equal to the asymptotic value of $\mathrm{D}$ at $\mathrm{I}=\overline{\boldsymbol{T}}$. Now, it is evident from (4.11) that there is an asymptotic expansion of $\mathrm{r}(\mathrm{t})$ in the form

$$
r(t) \sim t^{-a / b} \sum_{m=0}^{\infty} \sum_{n=0}^{\infty} r_{m n} t^{-m}\left(t^{a / b}\right)^{-n}
$$

and a corresponding expression for $\mathrm{D}(\mathrm{t})$ obtained from (4.19) by term-by-term integration. With (4.11) and (4.17) it is not difficult to see that the leading term expansion for $D(t)$ is

$$
D(t) \sim \frac{1}{f_{0}(a-b)}\left[\left\{\frac{c}{(1-1}\right\}^{1-b}\left(\frac{b c t}{1-b}\right)^{b-a}\right]^{1 / b} .
$$

We do not discuss further in this paper the technical details of this rather routine numerical method of solution, except to point out that the appropriate range of values of $\bar{T}$ for an accurate solution of our problem depends on the number of terms we keep in the asymptotic expansion for $D(t)$. The maximum consumption rate per head obtained by the above method is tabulated in Table 1 for different parameter values considered in [3] and [4]. In all cases, the elasticity of output with respect to the capital input, $a$, is fixed at 0.2 (and $f_{0}=1$ ). From the 
results in Table 1, we see that

(1) The effect of the unit resource extraction cost on the maximum consumption rate per head, $\boldsymbol{c}_{\max }$, is more substantial when the elasticity of output with respect to the resource input, $b$, is smaller.

(2) The effect of the size of the initial resource deposit on $c_{\max }$ is more substantial when $\boldsymbol{b}$ is larger.

(3) The effect of the size of the initial capital endowment on $\boldsymbol{c}_{\max }$ is more substantial when $b$ is smaller.

\section{Table 1}

Maximum Consumption Rate per Head for a

Single-Grade Resource $\left(a=0.2, f_{0}=1\right)$

\begin{tabular}{|c|c|c|c|c|c|}
\hline \multirow[b]{2}{*}{$b$} & \multirow[b]{2}{*}{$k_{0}$} & \multirow[b]{2}{*}{$D_{0}$} & \multicolumn{3}{|c|}{$c_{\max }$} \\
\hline & & & $\theta=0$ & $\boldsymbol{\theta}=0.03$ & $\theta=0.09$ \\
\hline \multirow[t]{9}{*}{0.05} & 1.2 & 10 & 0.99884 & 0.98228 & 0.95310 \\
\hline & & 25 & 1.04819 & 1.00717 & 0.95706 \\
\hline & & 50 & 1.08713 & 1.01348 & 0.95710 \\
\hline & 2.4 & 10 & 1.11436 & 1.10501 & 1.08708 \\
\hline & & 25 & 1.16942 & 1.14536 & 1.10525 \\
\hline & & 50 & 1.21287 & 1.16540 & 1.10742 \\
\hline & 4.8 & 10 & 1.24324 & 1.23801 & 1.22769 \\
\hline & & 25 & 1.30467 & 1.29103 & 1.26531 \\
\hline & & 50 & 1.35314 & 1.32531 & 1.27890 \\
\hline \multirow[t]{9}{*}{0.10} & 1.2 & 10 & 0.91842 & 0.90975 & 0.89148 \\
\hline & & 25 & 1.01685 & 0.99219 & 0.93979 \\
\hline & & 50 & 1.09826 & 1.04326 & 0.94802 \\
\hline & 2.4 & 10 & 0.99195 & 0.98731 & 0.97777 \\
\hline & & 25 & 1.09826 & 1.08524 & 1.05757 \\
\hline & & 50 & 1.18618 & 1.15743 & 1.09630 \\
\hline & 4.8 & 10 & 1.07136 & 1.06889 & 1.06386 \\
\hline & & 25 & 1.18618 & 1.17927 & 1.16491 \\
\hline & & 50 & 1.28115 & 1.26600 & 1.23372 \\
\hline \multirow[t]{9}{*}{0.15} & 1.2 & 10 & 0.76025 & 0.75798 & 0.75314 \\
\hline & & 25 & 0.89367 & 0.88678 & 0.87051 \\
\hline & & 50 & 1.00995 & 0.99349 & 0.94689 \\
\hline & 2.4 & 10 & 0.79188 & 0.79073 & 0.78834 \\
\hline & & 25 & 0.93086 & 0.92740 & 0.91987 \\
\hline & & 50 & 1.05198 & 1.04391 & 1.02481 \\
\hline & 4.8 & 10 & 0.82484 & 0.82426 & 0.82308 \\
\hline & & 25 & 0.96960 & 0.96786 & 0.96423 \\
\hline & & 50 & 1.09576 & 1.09175 & 1.08301 \\
\hline
\end{tabular}




\section{M aximum terminal capital stock for an unspecified planning period}

In preparation for a study of the two-grade resource problem investigated in [4], we consider in this section a finite-planning-period problem in which the level of consumption rate $\mathrm{c}$ per head is specified. The goal is to choose $\mathrm{r}(\mathrm{t})$ and the unspecified finite planning period $(0, T)$ so that $\mathrm{k}(\mathrm{T})$ is a maximum. The maximization is subject to the capital-accumulation rule (2.1), a prescribed finite-resource-stock condition (2.2), the initial capital endowment (2.3) and the nonnegativity of capital stock and resource flow (2.4). In the Mayer-type formulation of Sec. 3 with the state equation $\dot{\boldsymbol{c}} 0$ omitted (since $c$ is now a prescribed constant), the first-order necessary condition for this problem consists of the ODE (3.4) and (3.5), the inequality (3.7) and the auxiliary conditions (see [5])

$$
\begin{aligned}
\lambda_{k}(T) & =1, \\
\left\{\lambda_{k}[f(k, r)-\theta r-c]-\lambda_{D} r\right\}_{t=T} & =0 .
\end{aligned}
$$

Note that we must work with $\lambda_{\boldsymbol{k}}$ instead of $\boldsymbol{p}$ for this problem in view of (5.1).

For a Cobb-Douglas technology and an interior solution, we use the same procedure as in Sec. 4 to get the BVP

$$
\begin{aligned}
\dot{k} & =f_{0} k^{a} r^{b}-\theta r-c \quad k(0)=k_{0}, \\
\dot{r} & =-\frac{a c r}{\mathrm{k}(1-\mathrm{b})}[1-\bar{\theta} r], \\
\dot{D} & =-r, \quad D(0)=D_{1}, \quad D(T)=0
\end{aligned}
$$

(where we denote the total resource deposit for this problem by $D_{1}$ instead of $D_{0}$ ) and the equation

$$
\lambda_{k}\left[b f_{0} k^{a} r^{b-1}-\theta\right]=\lambda_{D}
$$

With the help of (5.6) and (5.1), the remaining condition (5.2) may be written as

$$
f_{0}(1-b) k^{a}(T) r^{b}(T)=c .
$$

Equations (5.3a),(5.4),(5.5a) and (5.6) constitute a third-order system of four equations for $\mathbf{k}(\mathbf{t}), \mathbf{r}(\mathbf{t}), \mathrm{D}(\mathbf{t})$, and $\lambda_{k}(t)$ with $\lambda_{D}$ and $T$ as unknown parameters. The five auxiliary conditions (5.1), (5.3b), (5.5b, c) and (5.7) determine $\lambda_{D}, T$ and the three constants of integration associated with the third-order system. For a solution of the above two-point boundary-value problem, we proceed as in Sec. 4 and form the first-order ODE (4.5) for $d k / d r$, which is then solved to get

$$
f_{0}(1-b) k^{a}(t) r^{b}(t)=\mathrm{c},
$$


where (5.7) was used to determine the constant of integration. Note that (5.8) is the equation for the "optimal trajectory" of Sec. 4. Thus the way to maximize the terminal capital stock for a finite unspecified planning period is to get on the "optimal trajectory" for the infinite-horizon problem of maximum constant consumption rate per head, whatever the unit resource extraction cost may be." Conversely, the "optimal trajectory" of Sec. 4 is in fact the path of maximum capital accumulation for the final choice of $c$, and the maximum constant consumption rate per head over an infinite horizon is achieved by maximizing the terminal stock of capital.

To continue the solution process, we now use (5.8) to eliminate $\boldsymbol{k}$ from (5.4) so that the resulting equation, along with $(5.5 \mathrm{a}, \mathrm{b}, \mathrm{c})$, determines $\boldsymbol{r}(\boldsymbol{t})$ and $\mathrm{D}(\mathrm{t})$. Equation (5.8) is used to get $\boldsymbol{k}(\boldsymbol{t})$ from $\boldsymbol{r}(\boldsymbol{t})$. The initial condition (5.3.b), $\boldsymbol{k}(\boldsymbol{O})=\boldsymbol{k}_{0}$, is then used to determine $\boldsymbol{T}$. Finally, with $\lambda_{\boldsymbol{k}}$ given in terms of $\boldsymbol{k}, \boldsymbol{r}$ and $\lambda_{D}$ by (5.6), the condition (5.1), $\lambda_{k}(T)=1$, determines the constant $\lambda_{D}$.

For the special case $8=0$, the above solution process gives

$$
k(t)=k_{0}\left[1+\frac{b c t}{k_{0}(1-b)}\right], \quad r(t)=r_{0}\left[1+\left.\frac{b c t}{k_{0}(1-b)}\right|^{-a / b},\right.
$$

where

$$
r_{0} \equiv r(0)=\left[\left.\frac{c}{f_{0} k_{0}^{a}(1-b)}\right|^{1 / b} .\right.
$$

The expressions (5.9) and (5.10) are exactly the same as those for the maximumc problem for an infinite horizon. Finally, Eqs. $(5.5 \mathrm{a}, \mathrm{b}, \mathrm{c})$ give

$$
D_{1}=\int_{0}^{T} r(t) d t=\frac{k_{0} r_{0}(1-b)}{c(a-b)}\left\{1-\left[1+\frac{b c T}{k_{0}(1-b)}\right]^{(b-a) / b}\right\}
$$

or

$$
T=\frac{k_{0}(1-b)}{b c}\left\{\left[1-\frac{D_{1} c(a-b)}{k_{0} r_{0}(1-b)}\right]^{-b /(a-b)}-{ }_{1}\right\}
$$

where $\boldsymbol{r}_{\mathbf{0}}$ is defined by (5.10). The terminal capital stock in this case is

$$
k_{T} \equiv k(T)=k_{0}\left\{1-(a-b) D_{1} k_{0}^{(a-b) / b}\left[\frac{f_{0}(1-b)}{c}\right]^{(1-b) / b}\right\}^{-b /(a-b)}
$$

For a sufficiently small value of $D_{1}$ (or $k_{0}$ or $1 / c$ ) so that

$$
(a-b) D_{1} k_{0}^{(a-b) / b}\left[\frac{f_{0}(1-b)}{c}\right]^{(1-b) / b}<1
$$

${ }^{5}$ This result was evident from the numerical results of [4] and is now proved conclusively. 
the resource deposit is exhausted in finite time and $k_{T}$ remains finite. If instead of (5.14) we have $D_{1}, k_{0}$ or $1 /$ c sufficiently large so that

$$
(a-b) D_{1} k_{0}^{(a-b) / b}\left[\frac{f_{0}(1-b)}{c}\right]^{(1-b) / b}>1,
$$

then the resource deposit cannot be exhausted by the prescribed consumption rate and the efficient program adopted. Given $\boldsymbol{D}_{\mathbf{1}}$ and $\boldsymbol{k}_{\mathbf{0}}$, there is a choice of c which our efficient program can sustain by exhausting the resource deposit over an infinite future.

A numerical scheme similar to that used in Sec. 4 is also needed here for the various time paths of the optimal solution for the $\boldsymbol{\theta} \neq \mathbf{0}$ case. However, a relation between $k_{T}$ and c analogous to (5.13) may be obtained for this case without solving the boundary-value problem. Note that (5.5a) and (5.8) may be used to transform the equation for capital accumulation, (5.3a), into

$$
\dot{k}=\frac{b c}{1-b}+\theta \dot{D}
$$

Upon integration and observing (5.3b), (5.5b) and (5.5c), we get

$$
\boldsymbol{k}(T) \equiv k_{T}=k_{0}+\frac{b c T}{1-b}+\theta[D(T)-D(0)]
$$

or

$$
\frac{b c T}{1-\mathrm{b}}=k_{T}-k_{0}+\theta D_{1}
$$

At the same time, Eq. (5.4) gives

$$
\frac{b c T}{1-b}=-\frac{b}{a}\left[\frac{c}{f_{0}(1-b)}\right]^{1 / a} \int_{r_{0}}^{r_{T}} \frac{d r}{r^{1+b / a}(1-\bar{\theta} r)},
$$

where $\boldsymbol{r}_{\mathbf{0}}$ is given by (5.10) and

$$
r_{T}\left[\frac{c}{f_{0}(1-b) k_{T}^{a}}\right]^{1 / b} .
$$

We may use (5.17) to eliminate $\boldsymbol{T}$ from (5.18) to get

$$
k_{T}-k_{0}+\theta D_{1}=-\frac{b}{a}\left[\frac{c}{f_{0}(1-b)}\right]^{1 / a} \int_{r_{0}}^{r_{T}\left(k_{T}\right)} \frac{d r}{r^{1+b / a}(1-\bar{\theta} r)}
$$

which is a relation for $k_{T}$ and c. Note that (5.20) becomes a trivial identity if $\boldsymbol{\theta}=0$. 
It should be noted that if $\boldsymbol{k}_{\mathbf{0}}$ is too small, so that $\boldsymbol{r}_{\mathbf{0}}$, as specified by (5.10) for all $8>0$, is greater than $D_{1}$, then it will not be possible to steer the economy onto the "optimal trajectory." Even if $r_{\mathbf{0}}<D_{1}$, so that we can get the economy on "the "optimal trajectory," the optimal program for the case $8>0$ actually calls for a gradual reduction of the initial capital stock if the prescribed $\mathrm{c}$ is so large that (cf. Appendix)

$$
k_{0}^{a}<\frac{1}{f_{0}}\left(\frac{\theta}{b}\right)^{b}\left(\frac{c}{1-b}\right)^{1-b}
$$

\section{A two-grade resource deposit}

Up to now, we have restricted our attention to the case of a homogeneous deposit of resource with the same unit extraction cost for the entire deposit. In reality, the entire stock of a single resource may consist of several deposits of different grades characterized by different unit extraction costs. To investigate the implications of extraction-cost differentials, a simple variation of the model of Sec. 4 was examined in [4]. In that paper, we suppose that the resource endowment consists of two grades only: a low-cost grade in amount $D_{1}$ with a unit extraction cost $\boldsymbol{\theta}_{1}$, and a high-cost grade in amount $D_{2}$ with a unit extraction $\operatorname{cost} \boldsymbol{\theta}_{\mathbf{2}}\left(>\boldsymbol{\theta}_{\mathbf{1}}\right)$. We know from an analysis in [4] that an infinite-horizon growth program cannot be optimal under the criterion of maximum constant consumption rate per head unless it exhausts the low-cost deposit first, in finite time, and then spreads the high-cost deposit over the infinite remaining future. Call the first part Phase I and the infinite tail Phase II. The switch over time $\boldsymbol{T}$ must be chosen to maximize the permanently sustainable constant level of consumption rate per head $\mathrm{c}$.

Suppose the low-cost deposit is exhausted at time $\boldsymbol{T}$. Then starting with time $T$, the economy has only a homogeneous single-grade (high-cost) resource left. The problem for Phase II is then one of maximizing the constant consumption rate per head c over an infinite planning period with a finite resource deposit $\boldsymbol{D}_{\mathbf{2}}$ and an initial stock of capital $\boldsymbol{k}_{\boldsymbol{T}}$ inherited from Phase I. This is a problem already solved in Sec. 4 , if we note that our economy evolves according to time elapsed and not calendar time, so that it is indifferent to a Phase II starting time at $t=T$ or $t=\mathrm{O}$. From the results of Sec. 4, the solution c for this problem is an increasing concave function of the initial capital stock $\boldsymbol{k}_{\boldsymbol{T}}$.

Now for Phase I, we have a homogeneous (low-cost) resource problem for a specified c and a finite but unspecified planning period $T$, with the deposit to be exhausted by the end of that planning period. The planning problem there is to adjust the planning period $\boldsymbol{T}$ and the resource extraction rate $\mathrm{r}(\mathrm{t})$ to maximize that terminal capital stock $k_{T}$ at the end of this period, since $k_{T}$ will be used as the intial capital stock for Phase II and a maximum $k_{T}$ will make the maximum sustainable c for Phase II as large as possible. This is a problem already solved in Sec. 5.

For the solution of the original two-grade resource problem, we must adjust the value of $\mathrm{c}$ in Phase I so that the resulting maximum $k_{T}$, when used as the 
initial capital stock for Phase II, gives the same value of $\mathrm{c}$ for the optimal solution of Phase II. From a mathematical viewpoint, the optimal solutions for $c$ and $\boldsymbol{k}_{\boldsymbol{T}}$ must be determined simultaneously. To be more specific, we have to solve the following three-point boundary-value problem with $\overline{\boldsymbol{\theta}}_{\boldsymbol{k}}=(\mathbf{1}-\boldsymbol{b}) \boldsymbol{\theta}_{\boldsymbol{k}} / \boldsymbol{b c}$ :

Phase I $(0 \leqslant t \leqslant T)$ :

$$
\begin{gathered}
(1-b) f_{0} k_{\mathrm{I}}^{a} r_{\mathrm{I}}^{b}=c, \quad \dot{r}_{\mathrm{I}}=-\frac{a c r_{\mathrm{I}}}{(1-b) k_{\mathrm{I}}}\left(1-\bar{\theta}_{1} r_{\mathrm{I}}\right), \quad \dot{D}_{\mathrm{I}}=-r_{\mathrm{I}} \\
k_{\mathrm{I}}(0)=k_{0}, \quad D_{\mathrm{I}}(0)=D_{1}, \quad D_{\mathrm{I}}(T)=0 .
\end{gathered}
$$

Phase $I I(T \leqslant t<o o)$ :

$$
\begin{gathered}
(1-b) f_{0} k_{\mathrm{II}}^{a} r_{\mathrm{II}}^{b}=c, \quad \dot{r}_{\mathrm{II}}=-\frac{a c r_{\mathrm{II}}}{(1-b) k_{\mathrm{II}}}\left(1-\bar{\theta}_{2} r_{\mathrm{II}}\right), \quad \dot{D}_{\mathrm{II}}=-r_{\mathrm{II}}, \\
k_{\mathrm{II}}(T)=k_{\mathrm{I}}(T), \quad D_{\mathrm{II}}(T)=D_{2}, \quad D_{\mathrm{II}}(\infty)=0 .
\end{gathered}
$$

To the extent that we have $\boldsymbol{\theta}_{2}>0$, a numerical procedure of the type described in Sec. 4 is necessary for handling the Phase II part of the problem. With a slight modification, the same numerical procedure also solves the entire two-grade resource problem. The modification consists of recalculating $k_{T}(c)$ by solving the boundary-value problem (6.1), (6.2) for $0<\mathrm{t}<\boldsymbol{T}$ for each new iterate $\mathrm{c}$ in the iterative scheme of Phase II (cf. Sec. 4). For $\boldsymbol{\theta}_{1}=0, \boldsymbol{k}_{T}(\boldsymbol{c})$ is easily determined by (5.13). For $\boldsymbol{\theta}_{1} \# 0$, we may use the integral relation (5.20) or solve the boundaryvalue problem (5.4), (5.5), (5.8) directly for $k_{T}(c)$.

The above numerical procedure has been used to obtain the solution of specific two-grade resource problems studied in [4] (with better accuracy) in about forty seconds of computer time on an IBM 360-168, which is several orders of magnitude less than that required by the search procedure used in [4]. For $\boldsymbol{\theta}_{1}=0$, the explicit expressions for $\boldsymbol{T}$ and $\boldsymbol{k}_{\boldsymbol{T}}$ given by (5.12) and (5.13) further reduce the computing time required for these problems. The new results

\section{Table 2}

Maximum Consumption Rate per Head and Switch Over Time for a Two Grade Resource Deposit $\left(\boldsymbol{a}=0.2, \boldsymbol{f}_{\mathbf{0}}=1, \boldsymbol{\theta}_{\mathbf{2}}=0.09\right)$

\begin{tabular}{rcccccccccccc}
\hline & & & & \multicolumn{4}{c}{$\mathrm{c}$} & \multicolumn{2}{c}{$\boldsymbol{k}_{\boldsymbol{T}}$} & \multicolumn{2}{c}{$\boldsymbol{T}$} \\
\cline { 6 - 12 } Case & $\boldsymbol{b}$ & $\boldsymbol{k}_{\mathbf{0}}$ & $\boldsymbol{D}_{\mathbf{1}}$ & $\boldsymbol{D}_{\mathbf{2}}$ & $\boldsymbol{\theta}_{\mathbf{1}}$ & $=0.0$ & \multicolumn{2}{c}{$\boldsymbol{\theta}_{\mathbf{1}}=\mathbf{0 . 0 3} \boldsymbol{\theta}_{\mathbf{1}}=\mathrm{O} . \mathrm{O}$} & $\boldsymbol{\theta}_{\mathbf{1}}=\mathbf{0 . 0 3}$ & $\boldsymbol{\theta}_{\mathbf{1}}=\mathbf{0 . 0}$ & $\boldsymbol{\theta}_{\mathbf{1}}=\mathbf{0 . 0 3}$ \\
\hline I & 0.05 & 2.4 & 10 & 50 & 1.1587 & 1.1406 & 2.9779 & 2.7621 & 9.4743 & 11.030 \\
II & 0.10 & 2.4 & 10 & 50 & 1.1560 & 1.1386 & 3.2100 & 2.9446 & 6.3022 & 6.6761 \\
III & 0.15 & 2.4 & 10 & 50 & 1.0743 & 1.0675 & 4.3140 & 3.9520 & 10.537 & 9.8312 \\
IV & 0.05 & 2.4 & 10 & 25 & 1.1566 & 1.1392 & 3.0097 & 2.7889 & 10.023 & 11.489 \\
V & 0.10 & 2.4 & 10 & 25 & 1.1209 & 1.1080 & 3.5976 & 3.3083 & 9.6156 & 9.8150 \\
VI & 0.05 & 2.4 & 50 & 50 & 1.2291 & 1.1693 & 3.9545 & 3.1102 & 24.057 & 35.914 \\
VII & 0.05 & 4.8 & 10 & 50 & 1.3154 & 1.3036 & 5.5193 & 5.2771 & 10.389 & 11.325 \\
VIII & 0.10 & 4.8 & 10 & 50 & 1.2764 & 1.2675 & 6.0520 & 5.7652 & 8.8408 & 8.9832 \\
IX & 0.15 & 2.4 & 10 & 25 & 0.98496 & 0.98053 & 6.7177 & 6.2552 & 24.841 & 24.014 \\
\hline
\end{tabular}


for the cases studied in [4] and the corresponding solutions for $\boldsymbol{\theta}_{\mathbf{1}}=\mathbf{0}$ are summarized in Table 2. These results show that the reduction in the unit resource extraction cost allows a significant increase in $c_{\max }$ only for case VI, which has a relatively large initial resource endowment. (A similar significant increase also occurs for another run with the same input parameters as VI but $\boldsymbol{k}_{\mathbf{0}}=4.8$.)

\section{Concluding remarks}

The developments in the preceding sections offer a new perspective on R. M. Solow's maximum-sustainable-consumption growth theory when an exhaustible resource is essential to production. The new control-theoretic formulation enables us to develop new efficient deductive methods of solution for obtaining the maximum sustainable consumption rate and other relevant information for both a single-grade and a two-grade resource deposit problem. It also enables us to gain a new insight to the economic content of the relevant optimal growth paths. While the method for two-grade deposit problems can be extended to handle multigrade deposits, a different method of solution particularly attractive for problems with three (or more) grades will be discussed in [6], where results for specific three-grade deposit problems will be reported and analyzed.

\section{A ppendix. Trajectories in the resource-capital space}

For a constant 8 and a fixed c, the autonomous system (4.1) and (4.4), written as

$$
\dot{k}=f_{0} k^{a} r^{b}-\theta r-c, \quad \dot{r}=-\frac{a c r}{(1-b) k}(1-\bar{\theta} r)
$$

where $\bar{\theta}=\theta(1-b) / b c$, has one critical point $(\bar{r}, \bar{k})$ in the first quadrant of the $r, \mathrm{k}$-plane. This unstable improper node is located at the intersection of the curve

$$
f_{0} \bar{k}^{a} \bar{r}^{b}-\theta \bar{r}-c=0
$$

and the line $\bar{r}=1 / \bar{\theta}=b c / \theta(1-b)$ (see Fig. 1). With the change of variable $\boldsymbol{u}=1 / \mathrm{k}$, it is not difficult to see that all points on the $\boldsymbol{u}=0$ line are also critical points (see Fig. 2). Along the vertical line $\mathrm{r}=\overline{\boldsymbol{r}}=1 / \bar{\theta}$, the direction of the trajectories is vertical upward for $k>\bar{k}$ and vertical downward for $\boldsymbol{k}<\bar{k}$. Along the curve (A.2) (the dashed curve in Fig. 1), the direction of the trajectories is horizontal to the left for $r<\bar{r}$ and horizontal to the right for $r>\bar{r}$. The locus of horizontal slopes (A.2) and the vertical line $r=\bar{r}$ divide the first quadrant of the $\mathrm{r}$,k-plane into four separate regions (see Fig. 1). All trajectories in the lower right region tend to $(\infty, 0)$, while those in the lower left region tend to $(0,0)$. The situation in the two upper regions is more complicated. In the upper left region, those trajectories sufficiently close to the dashed curve will eventually reach that curve with horizontal slope and then turn downward toward $(0,0)$, while those away from the dashed curve (but still to the left of $r=\bar{r}$ ) will head toward a nonisolated critical point on the critical line $(\mathrm{r}, \mathrm{u}=0)$ as shown in Fig. 2. 


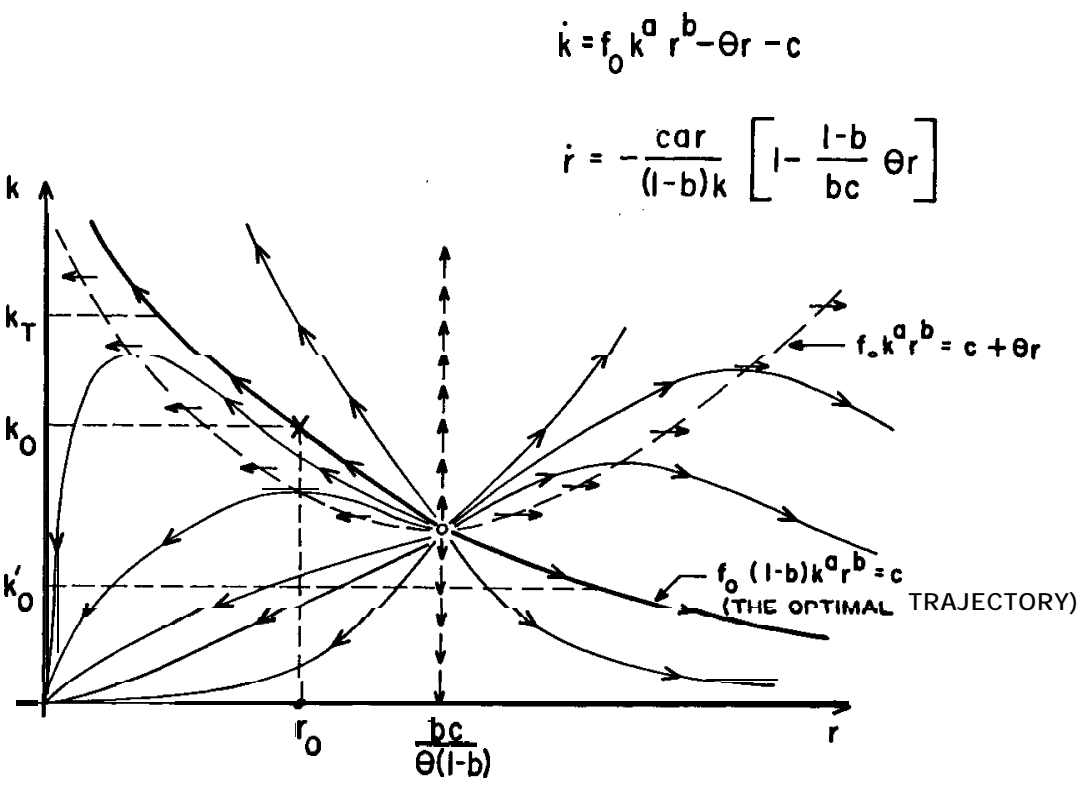

Figure 1. Trajectories in the space of capital stock per head versus resource extraction rate per head.

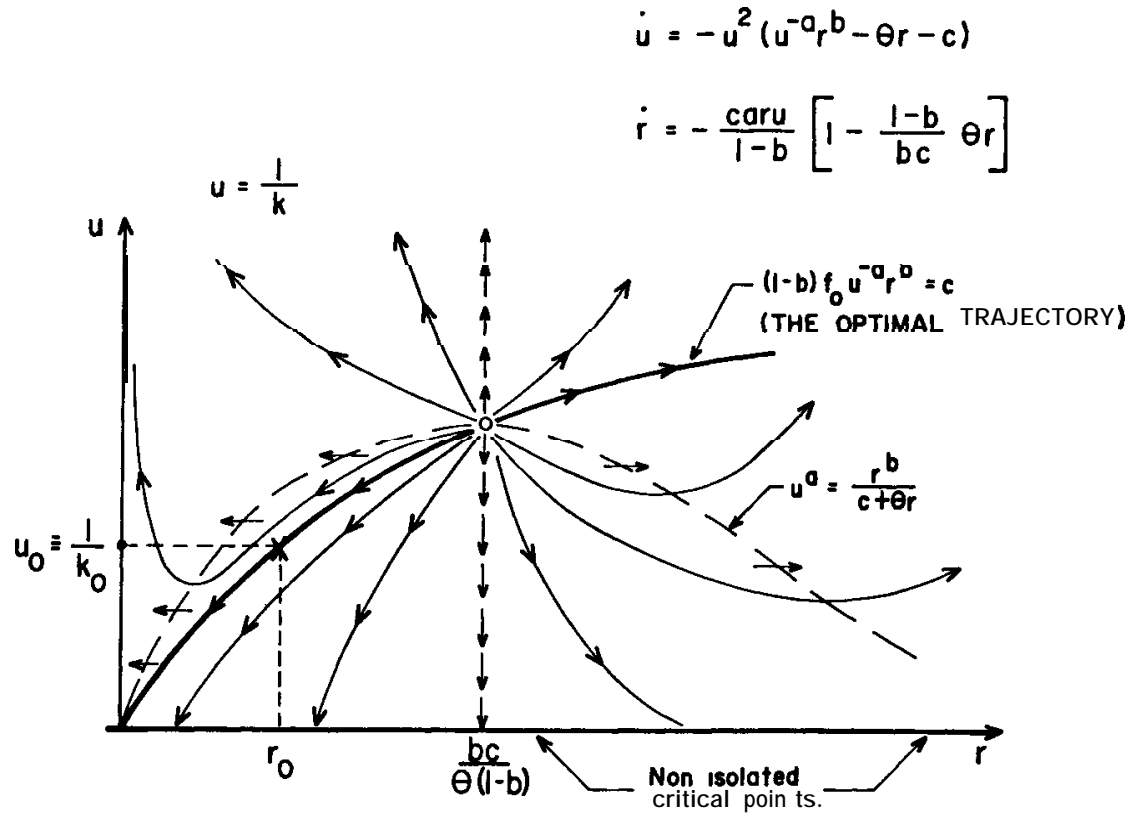

Figure 2. Trajectories in the space of capital labor stock per head versus resource extraction rate per head. 
Evidently, there is a trajectory dividing these two groups of trajectories; it tends to the critical point $(0, \infty)$ [or $(0,0)$ in the $r$, $u$-plane] as t tends to infinity. This is the separatrix

$$
f_{0}(1-b) k^{a} r^{b}=c
$$

which we have called the "optimal trajectory" and have indicated by a heavy curve in Figs. 1 and 2 . It can be verified that, geometrically, the optimal trajectory curve (A.3) passes through the critical point $(\bar{r}, \bar{k}) .^{\mathbf{6}}$ In the upper right-hand comer, trajectories close to the horizontal-slope locus will eventually be bent over and head toward $(\infty, 0)$, while those away from the horizontal-slope locus will head toward a point on the critical line $(r, \infty)$ (see also Fig. 2).

For the infinite-horizon single-grade resource problem of Sec. 4, we are only interested in the trajectories to the left of the vertical line $\boldsymbol{r}=\overline{\boldsymbol{r}}$, since the other trajectories exhaust the finite resource deposit in finite time and there will be no production, and therefore no consumption, thereafter. The same reason also eliminates all trajectories in the upper left region to the right of the "optimal trajectory." Finally, a trajectory to the left and below the "optimal trajectory" cannot be a solution, since such a trajectory exhaust the capital stock in finite time and there will be no production, and therefore no consumption, thereafter. This leaves the "optimal trajectory" as the only solution candidate for the optimal-control problem. Given an initial stock of capital $\boldsymbol{k}_{\mathbf{0}}$ and a resource deposit $D_{0}$, the optimal program consists of picking an initial resource flow rate $\boldsymbol{r}_{\mathbf{0}}$ according to (A.3) to get on the "optimal trajectory" and then adjusting the consumption rate $\mathrm{c}$ so that the resource deposit is completely used up over the planning period, but not before that. Adjusting $\mathrm{c}$ amounts to a horizontal shift of the vertical line $\boldsymbol{r}=\overline{\boldsymbol{r}} \equiv \boldsymbol{b c} \boldsymbol{\theta} \boldsymbol{\theta}(\mathbf{1}-b)$. For a fixed $\mathrm{c}$, changing the unit extraction cost 8 has the same effect of shifting the vertical line $r=\bar{r}$ horizontally. As 8 tends to zero, the vertical line moves further and further to the right, eventually out of sight, and we recover the situation studied in [3].

For the finite-horizon problem of maximum terminal capital stock (Sec. 5)-as well as phase I of the two-grade resource problem (Sec. 6)--the consumption rate per head $\mathrm{c}$ is prescribed, and it is not possible to get on the northwest-corner portion of the optimal trajectory if $k_{0}<\bar{k}$. Nevertheless, the results of Sec. 5 imply that the maximum terminal capital is still attained along the (southeast-corner portion of the) optimal trajectory for this case, even though this optimal program actually calls for a continual decrease of the capital stock.

\section{Acknowledgments}

The author gratefully acknowledges the help of his colleague, Professor Uri Ascher of the Department of Computer Science at U.B.C., and his student assistants, John Flinn and David Rollin, on the computational aspects of the research project.

${ }^{6}$ Of course, the curve is the union of two different trajectories eminating from the critical point but heading in the opposite directions. 


\section{R eferences}

1. R. M. SOLOW, The economics of resources or the resources of economics, the Richard T. Ely Lecture, J. Amer. Econ. Assoc. 64 (2): 1-14 (May 1974).

2. J. RAWLS, A Theory of Justice, Belknap (Harvard U. P.), Cambridge, Mass., 1971.

3. R. M. SOLOW, Intergenerational equity and exhaustible resources, Rev. Econom. Studies (Symposium Issue), 1974, pp. 29-46.

4. R. M. SOLOW and F. Y. M. WAN, Extraction costs in the theory of exhaustible resources, Bell J. Econom. 7:359-370 (1976).

5. A. E. Bryson and Y. C. Ho, Applied OptimaI Control, Ginn, Waltham, Mass., 1969.

6. U. ASCHER and F. Y. M. WAN, Numerical solution for maximum sustainable consumption growth with a multi-grade exhaustible resource, SIAM J. Sci. Statis. Computing 1 (to be published, 1980).

UNIVERSITY OF BRITISH COLUMBIA

(Received March 16, 1979) 